\title{
New Onset Heart Failure Presents with Advanced, Global Cardiac Remodeling when Associated with Atrial Fibrillation
}

\author{
Vinay Mehta ${ }^{1}$, Alexander Albers ${ }^{1}$, Maharaj Singh ${ }^{2}$, and Timothy Paterick ${ }^{1}$ \\ ${ }^{1}$ Aurora BayCare Medical Center \\ ${ }^{2}$ Aurora Health Care
}

June 3, 2020

\begin{abstract}
Introduction: This study aimed to evaluate the extent of baseline cardiac remodeling present in patients with new-onset cardiomyopathy $(\mathrm{CM})$ with and without atrial fibrillation $(\mathrm{AF})$. AF concurrent with heart failure is associated with increased morbidity and mortality. There is limited research comparing the extent of cardiac remodeling present in patients with newonset CM associated with AF (AF-CM group) to that present in patients with new-onset CM without any concomitant or antecedent dysrhythmia (CM group). Methods and Results: Patients with a left ventricular ejection fraction [?] $40 \%$ without a prior history of CM were identified from our healthcare system's electronic medical records for the period of January 1, 2012 to September 30, 2016. Those with an antecedent or concomitant diagnosis of AF comprised the AF-CM group, and those with no prior dysrhythmia comprised the CM group. The AF-CM group ( $\mathrm{n}=196)$ was compared with the CM group (n=197). The groups were controlled for left ventricular ejection fraction. Patients in the AF-CM group had more left atrial, right atrial, and right ventricular remodeling, more advanced right ventricular systolic dysfunction, and increased severity of both tricuspid and mitral regurgitation at presentation than the CM group. Conclusion: Patients with new-onset CM with AF had greater global cardiac remodeling at presentation than those with $\mathrm{CM}$ not associated with AF.
\end{abstract}

\section{Introduction}

This study evaluated the morphological and physiological differences between new-onset cardiomyopathy $(\mathrm{CM})$ with and without atrial fibrillation (AF). New-onset CM was defined as left ventricular ejection fraction (LVEF) [?] $40 \%$ with no prior history of CM. The distinction between the two CM groups is whether there was concomitant or antecedent AF (AF-CM group) or no history of dysrhythmia (CM group). There is scant literature comparing the degree of cardiac remodeling present at baseline between these two groups that are commonly encountered in clinical practice.

\section{Methods}

This study was approved by the local Aurora Institutional Review Board.

Study population and data collection

We identified patients with new-onset CM, defined as an LVEF [?]40\% and no prior history of low EF, from January 1, 2012 to September 30, 2016 through retrospective review of data from electronic medical records (EPIC, Verona, WI). Both inpatients and outpatients were included in the study. Patients with any pre-existing evidence of CM based on office notes and/or cardiac imaging were excluded. Patients with a pre-existing cardiovascular implantable electronic device or with a history of any other dysrhythmia, including frequent premature ventricular contractions were excluded, as were patients with an unreadable or poor-quality echocardiogram, mortality during inpatient admission, or congenital heart disease. Patients were divided into two groups: the AF-CM group encompassed those with an antecedent or concomitant 
diagnosis of $\mathrm{AF}$, and the $\mathrm{CM}$ group comprised those with no history of preceding dysrhythmia. Patients in the CM group had not had any atrial or ventricular dysrhythmia in the 1 year preceding or the consecutive 2 months following the diagnosis of CM. Echocardiographic data were collected from the echocardiographic reports. Data were collected on both right atrial (RA) and left atrial (LA) size, LA volume, LA volume index, right ventricular (RV) size and systolic function, LV size, and presence and severity of both mitral and tricuspid regurgitation. Information about the treatment these patients received both as an inpatient and outpatient and outcome data also were collected. An almost equal number of patients were included for each $5 \%$ difference in LVEF. An equal number of patients were collected for LVEF <20\% (Figure 1). The demographic and clinical characteristics of these patients are listed in Table 1.

\section{Statistical methods}

All categorical and continuous variables were described using appropriate descriptive statistics. For example, all categorical variables, such as sex, race, tobacco and alcohol use, hypertension, history of percutaneous coronary intervention, hyperlipidemia, coronary artery disease, diabetes mellitus, history of drug abuse, outpatient diagnoses (congestive heart failure, myocardial infraction), mortality, and categories of LVEF, were described as frequencies and percentages, and wherever appropriate chi-square and/or Fisher's exact test were used to compare the AF-CM and CM groups. All continuous variables, such as age, body mass index, LVEF, and LV diastolic diameter were described as mean \pm standard deviation, and the mean values of these variables for the two groups were compared using t test for independent samples. An alpha of 0.05 was used for all statistical tests, and all statistical analysis was done using SAS 9.4 version, SAS Institute, Cary, NC.

\section{Results}

(Figure 2, Table 2)

\section{Left ventricle}

Both the AF-CM group and CM group were controlled for presenting LVEF. Despite having heart failure with reduced EF, LV dimensions were within normal limits for most patients in both groups. LV cavity size was slightly larger in the isolated CM group, with a median value of $54.5 \mathrm{~mm}$ vs $51.9 \mathrm{~mm}$ in the AF-CM group $(\mathrm{p}=0.0024)$. There were also more patients with moderately to severely increased LV size at presentation in the isolated CM group $(45 / 184,24.45 \%)$ than in the AF-CM group $(25 / 188,13.29 \%)$ (Figure 3A). The LV was the only cardiac chamber that showed greater remodeling at baseline in the isolated CM group.

\section{Right ventricle}

Most patients in the CM group $(134 / 193,69.43 \%)$ had a normal RV size at presentation, whereas a majority of patients in the AF-CM group $(112 / 192,58.33 \%$; $\mathrm{p}<0.001)$ had an enlarged RV at the time of the initial diagnosis of CM. Almost a quarter of patients in the AF-CM group had moderate-to-severe RV enlargement on the initial echocardiogram, whereas $<10 \%$ of patients had a similar degree of enlargement in the CM group. RV systolic dysfunction was much more prevalent in the AF-CM group. More than half of patients in the CM group $(114 / 194,58.76 \%)$ had a normally functioning RV, whereas a majority of patients in the AF-CM $(126 / 192,65.62 \%)$ group had RV dysfunction present at the time of the initial diagnosis $(\mathrm{p}<0.001)$. Moderate-to-severe RV dysfunction was more than twice as prevalent in the AF-CM (60/192, 31.2\%) group at presentation than in the CM group $(28 / 194,14.4 \%$; p <0.0001) (Figure 3B).

Left atrium

Greater LA enlargement was seen in the AF-CM group; mean LA volume index was severely enlarged in the AF-CM group $\left(51.93 \mathrm{~mL} / \mathrm{m}^{2}\right)$ vs only mildly enlarged $\left(38.52 \mathrm{~mL} / \mathrm{m}^{2}\right)$ in the CM group. A majority of patients $(92 / 161,57.1 \%)$ in the CM group had a normal or slightly enlarged LA, whereas only a small number of patients in the AF-CM group $(35 / 142,24.6 \%, \mathrm{p}<0.0001)$ had similar LA size. More than three-quarters of patients in the AF-CM group (107/142, 75.4\%) had moderate-to-severe LA enlargement at presentation. 
The percentage of patients with severe LA enlargement in the AF-CM group was almost twice (65.6\%) that of the isolated CM group $(34.4 \%, \mathrm{p}<0.0001)$ (Figure $3 \mathrm{~A}$ ).

Right atrium

RA remodeling was even more pronounced at baseline in the AF-CM group than the CM group. A majority of patients had a normal RA size in the isolated CM group (112/193, 58\%), while less than one-third of that number $(33 / 192,17.18 \%$ ) had a normal RA size in the AF-CM group ( $<<0.0001)$. Moderate-to-severe RA enlargement was prevalent in the AF-CM group (115/192, 59.9\%) while being relatively uncommon in the CM group $(31 / 193,16.1 \%)$ (Figure 3B).

Greater prevalence, severity of valvular regurgitation in AF-CM group

A majority of patients in the isolated CM group were reported to have either no or mild mitral regurgitation, $(119 / 176,67.6 \%)$, whereas almost half the patients in the AF-CM group (90/182, 49.4\%; p = 0.0010) had either moderate or severe mitral regurgitation. The difference in tricuspid regurgitation was even more marked; there was either no or just mild tricuspid regurgitation in the CM group for most patients (132/172, $76.7 \%)$, with less than a quarter $(40 / 172,23.3 \%)$ reported as having moderate-to-severe tricuspid regurgitation. On the other hand, in the AF-CM group more than twice that percentage of patients $(88 / 181,48.6 \%)$ were reported to have moderate-to-severe tricuspid regurgitation at presentation $(\mathrm{p}<0.0001)$ (Figure 4$)$.

AF-CM group more likely to present in the outpatient setting

Despite having markedly more advanced global cardiac remodeling at presentation, the AF-CM group patients were more likely to present and be diagnosed as outpatients. AF symptoms were much more likely to be atypical (tiredness, fatigue, weight gain) rather than typical (palpitations) in this group.

Mortality in the AF-CM group

The mortality rate in the AF-CM group was considerably higher than in the isolated CM group (Table 2). This was despite the CM group having a more acute presentation and greater elevation in traditional cardiac biomarkers. The Kaplan-Meier survival curves for the two groups diverged further apart throughout the time follow-up data were collected.

\section{Discussion}

Left ventricular size

Mean LV size was within normal parameters in both groups, though the dimensions were slightly larger in the isolated CM group. There were also more patients with moderate-to-severe enlargement in the CM group than the AF-CM group (Figure 3A). This may indicate that:

1. Patients in both groups had new-onset CM, given that, despite a low LVEF, the LV did not have a chance to significantly remodel in a majority of patients as increased LV size occurs progressively in both ischemic and nonischemic CM. ${ }^{1}$

2. That an LV myopathic process likely caused isolated LV dysfunction in the CM group, and a global pan-myocardial maladaptive process occurred in the AF-CM group that affected all chambers of the heart rather than just the LV. The only chamber that was more remodeled in the CM group was the $\mathrm{LV}$, pointing toward the underlying pathophysiological process having been more localized to the LV in the CM group.

Comparing biatrial remodeling in AF-CM and cm groups

There was notably more LA and RA remodeling present at baseline in the AF-CM group than the CM group. A larger LA portends an increased risk for development of $\mathrm{AF}^{2,3} \mathrm{AF}$ can also lead to enlargement of both the LA and RA. ${ }^{4}$ The triggers for AF have been localized to the LA in a majority of patients, ${ }^{5}$ but it is interesting to note that the difference in RA enlargement was greater in the AF-CM group. A large percentage of patients $(83.9 \%)$ in the CM group had a normal or mildly enlarged RA, whereas a majority 
of patients (59.9\%) in the AF-CM group had moderate-to-severe enlargement of the RA (Figure 3A). The more pronounced RA enlargement may be explained by:

1. RV enlargement/dysfunction and tricuspid regurgitation, both of which were more prevalent and severe in the AF-CM group, could have resulted in more advanced remodeling of the RA. ${ }^{6}$

2. Significant LA enlargement has been shown to occur in patients with heart failure with reduced EF. ${ }^{7}$ As isolated CM appears to be more of a localized LV myopathic process with increased LV remodeling present at baseline, it could have led to more adverse hemodynamic effects and remodeling on the LA in the CM group. This, in turn, could have resulted in a smaller relative difference in the size of the LA and a larger relative difference in the size of the right atrium between the two groups.

However, AF is known to cause both RA and LA enlargement. ${ }^{4}$ Biatrial enlargement could have caused both tricuspid and mitral annular dilation and functional tricuspid and mitral valve regurgitation, setting up a mutually reinforcing vicious remodeling effect with further enlargement of both atria, perpetuation of AF, and development of further ventricular remodeling and dysfunction (Figure 5). In addition, risk factors like hypertension and coronary artery disease, which were more prevalent in the AF-CM group, are also known to cause cardiac remodeling. ${ }^{8,9}$ But it does appear that the process of global, advanced remodeling transpired slowly though inexorably, maybe over months or even years, for the following reasons:

1. This degree of cardiac remodeling is unlikely to occur over hours or days even in the presence of serious underlying pathology.

2. Most of the patients in the AF-CM group presented with subacute or atypical symptoms, which is suggestive of the fact that the homeostatic mechanisms in these individuals had time to adapt to the profound structural and functional changes occurring in the heart.

Right ventricular remodeling and dysfunction

The degree and extent of RV enlargement and dysfunction was even greater in the AF-CM group. The exact pathophysiologic mechanisms for this are unknown. Possible reasons may include:

1. AF leads to RA enlargement, which in turn can cause tricuspid annular dilation and functional tricuspid regurgitation; ${ }^{6,10}$ the increased load due to tricuspid regurgitation can lead to RV enlargement and dysfunction (Figure 5).

2. The underlying pathophysiologic factors that increase the risk for development of AF, like obstructive sleep apnea, ${ }^{11}$ have also been implicated to cause RV dilation and dysfunction. ${ }^{12}$

3. Isolated CM being a more LV-centric disease process preferentially involves the $\mathrm{LV}$, with other chambers eventually getting involved due to LV dysfunction, whereas AF-CM, being a more systemic disease process, more uniformly effects all chambers, and thus the RV is in a more advanced disease state at presentation.

The answer remains perplexing: Nonetheless, significantly more advanced right-sided chamber involvement appears to be a characteristic phenotypical feature of AF-CM (Figures 2, 4).

Presence of coronary artery disease and biventricular dysfunction

Based on previous studies, biventricular dysfunction is more likely to be associated with nonischemic CM, whereas isolated LV dysfunction with wall motion abnormalities is more commonly encountered in patients with coronary artery disease or ischemic CM. ${ }^{13,14}$ In this study, the AF-CM group, despite having a higher prevalence of coronary artery disease (Table 2), had more biventricular cardiac dysfunction. Patients with biventricular remodeling/enlargement have also been found to be more symptomatic at presentation, ${ }^{15}$ but even though the AF-CM group patients had more marked biventricular dysfunction, they were less acutely symptomatic than the CM group patients. This again points toward the fact that this phenotype of CM likely develops more gradually, leading to a subacute and atypical presentation despite the presence of significantly more biventricular dysfunction. The subacute presentation, though, did not provide any protection against a worse prognosis: Patients in the AF-CM group had a higher mortality rate on follow-up. 
Differences in valvular regurgitation between the two groups

\section{Tricuspid Regurgitation}

The presence and severity of tricuspid regurgitation was greater in the AF-CM group, with almost twice the number having moderate-to-severe tricuspid regurgitation compared to the isolated CM group (Figures 2, 4). Postulated explanations include:

1. The relative difference in RA enlargement was even more marked between the two groups than LA enlargement. This may have led to relatively greater tricuspid annular dilation and functional tricuspid regurgitation. ${ }^{6,10}$

2. Factors like obstructive sleep apnea and obesity probably caused more baseline RV enlargement, tricuspid annular dilation, and tricuspid regurgitation.

3. Arrhythmia-induced and arrhythmia-mediated CMs do cause profound changes in cellular and extracellular matrix in the entire heart. ${ }^{16}$ This can cause more pan-myocardial chamber remodeling, rather than isolated LV remodeling, in AF-CM, leading to more severe tricuspid regurgitation.

\section{Mitral Regurgitation}

The prevalence of moderate-to-severe mitral regurgitation was also greater in the AF-CM group (49.4\% vs $32.4 \%$ ). There is increasing recognition that AF may be an etiological factor for mitral regurgitation. ${ }^{17}$ The difference between the two groups was less for mitral regurgitation than for tricuspid regurgitation. This may be owing to the heterogeneous remodeling of left-sided chambers seen in the two groups. While the right-sided chambers were uniformly more enlarged in the AF-CM group (Figure 3B), the same was not seen in the left-sided chambers (Figure 3A). The LA was significantly more remodeled in the AF-CM group, whereas the LV was slightly larger in the CM group. Both LA and LV dilation probably lead to functional mitral regurgitation. ${ }^{18}$ The fact that these factors were contradictory in these two groups may have led to a smaller relative difference in the degree of significant mitral regurgitation between them.

Generally, more severe valvular regurgitation is associated with a more symptomatic presentation. ${ }^{19-21}$ However, patients in the AF-CM group, despite having worse valvular regurgitation, had a more subacute presentation than those in the CM group, which again points toward a slow but unremitting pathophysiologic process set in motion in the AF-CM group.

Ubiquitous and advanced cardiac remodeling at presentation in the AF-CM group: A distinct phenotype of CM?

Even though a lot of research into arrhythmia-induced and arrhythmia-mediated CM has focused on the LV, the process of remodeling in AF-CM appears to be systemic and pan-cardiac. There are multiple mechanisms by which dysrhythmias have been proposed to cause CM, which involve complex and pervasive changes at the structural, functional, molecular, and electrophysiological levels. ${ }^{16,22}$ These changes affect the entire heart and not just the LV, and thus a more pan-myocardial pathology at presentation would be expected; this is what was found in the current study. An individual can also be predisposed to AF owing to a variety of risk factors, including older age, increased body mass index, and history of hypertension, among others. ${ }^{23}$ All these factors were more common in the AF-CM group, and it is possible that these factors independently caused some of the cardiac remodeling seen in this phenotype (Figure 5). Regardless of the temporal association of $\mathrm{AF}$ in the development of this disease, the AF-CM group phenotype appears to be quite distinctive from the CM group.

Limitations of the study

This is a retrospective study with all the limitations inherent in that type of study, including incomplete or inaccurate data and the possibility of selection bias. Echocardiographic reports were utilized for assessing LVEF; sometimes, these can be challenging to read, especially in patients with atrial dysrhythmias. One of the reasons a cut-off of $40 \%$ was selected was to minimize the probability of patients with a normal EF being included in the study. 
Each patient chart was thoroughly evaluated by a research coordinator to identify a group of new-onset $\mathrm{CM}$ patients with a concomitant or antecedent diagnosis of AF (AF-CM group); the CM group patients were also carefully assessed to rule out contribution of any dysrhythmias to CM. Nonetheless, it is possible that some CM group patients had AF that was undiagnosed or not captured in medical documentation or electrocardiographic, telemetry, or rhythm strip evaluation. We excluded patients with a cardiovascular implantable electronic device because a significant percentage of them have structural heart disease, and also, pacing can confound both patient characteristics and outcomes. Thus, we obtained as uncontaminated a sample as possible so that patients with AF-CM and CM without any dysrhythmias could be compared.

There were some missing data in reports; for example, some reports did not have LA or RA size listed. But the amount of missing data was low and a majority of data points were available in the reports.

Echocardiograms were read by different readers, had varying quality, and were performed on different echocardiography machines. Nonetheless, there is consistency in the broad narrative across all those studies and readers. Moreover, the likelihood of researcher bias in those echocardiography readers would be non-existent as they were not trying to accomplish a particular research goal, but were reading the studies in service of clinical decision-making in the real world.

\section{Conclusions}

AF-CM and isolated CM groups are frequently encountered in clinical practice. To our knowledge, this is the first study evaluating the differences in structural and functional cardiac remodeling at presentation in patients with new-onset CM, with and without AF. Despite having significantly greater cardiac remodeling at baseline, a majority of patients in the AF-CM group presented with atypical symptoms of AF and had less acutely perceptible congestive heart failure symptoms, yet had an inferior prognosis. The underlying pathophysiologic mechanisms for the development of AF-CM may be a complex interplay of comorbid conditions, atrial fibrillation itself, and the gradual progression of cardiac remodeling. This may set up a vicious, self-perpetuating pathophysiological cycle (Figure 5) that is relentless in its progression, leading to the development of a distinct phenotype of CM (Figure 2). Further studies to explore the distinctive characteristics, underlying pathophysiological mechanisms and appropriate treatment pathways for the AF-CM group will shed more light on this 21st-century "epidemic of cardiovascular disease."

\section{References}

1. Konstam MA, Kramer DG, Patel AR, Maron MS, Udelson JE. Left ventricular remodeling in heart failure: current concepts in clinical significance and assessment. JACC Cardiovasc Imaging 2011;4:98-108.

2. Vaziri SM, Larson MG, Benjamin EJ, Levy D. Echocardiographic predictors of nonrheumatic atrial fibrillation. The Framingham Heart Study. Circulation 1994;89:724-30.

3. Habibi M, Samiei S, Ambale Venkatesh B, et al. Cardiac magnetic resonance-measured left atrial volume and function and incident atrial fibrillation: Results from MESA (Multi-Ethnic Study of Atherosclerosis). Circ Cardiovasc Imaging 2016;9pii:e004299.

4. Sanfilippo AJ, Abascal VM, Sheehan M, et al. Atrial enlargement as a consequence of atrial fibrillation. A prospective echocardiographic study. Circulation 1990;82:792-7.

5. Haissaguerre M, Jais P, Shah DC, et al. Spontaneous initiation of atrial fibrillation by ectopic beats originating in the pulmonary veins. N Engl J Med 1998;339:659-66.

6. Najib MQ, Vinales KL, Vittala SS, Challa S, Lee HR, Chaliki HP. Predictors for the development of severe tricuspid regurgitation with anatomically normal valve in patients with atrial fibrillation. Echocardiography 2012;29:140-6.

7. Melenovsky V, Hwang SJ, Redfield MM, Zakeri R, Lin G, Borlaug BA. Left atrial remodeling and function in advanced heart failure with preserved or reduced ejection fraction. Circ Heart Fail 2015;8:295-303.

8. Nadruz W. Myocardial remodeling in hypertension. J Hum Hypertens 2015;29:1-6. 
9. Heusch G, Libby P, Gersh B, et al. Cardiovascular remodelling in coronary artery disease and heart failure. Lancet 2014;383:1933-43.

10. Utsunomiya H, Itabashi Y, Mihara H, et al. Functional tricuspid regurgitation caused by chronic atrial fibrillation: A real-time 3-dimensional transesophageal echocardiography study. Circ Cardiovasc Imaging 2017;10pii:e004897.

11. Linz D, McEvoy RD, Cowie MR, et al. Associations of obstructive sleep apnea with atrial fibrillation and continuous positive airway pressure treatment: A review. JAMA Cardiol 2018;3:532-40.

12. Maripov A, Mamazhakypov A, Sartmyrzaeva M, et al. Right ventricular remodeling and dysfunction in obstructive sleep apnea: A systematic review of the literature and meta-analysis. Can Respir J 2017;2017:1587865.

13. Iskandrian AS, Helfeld H, Lemlek J, Lee J, Iskandrian B, Heo J. Differentiation between primary dilated cardiomyopathy and ischemic cardiomyopathy based on right ventricular performance. Am Heart J 1992;123:768-73.

14. La Vecchia L, Zanolla L, Varotto L, et al. Reduced right ventricular ejection fraction as a marker for idiopathic dilated cardiomyopathy compared with ischemic left ventricular dysfunction. Am Heart J 2001;142:181-9.

15. La Vecchia L, Paccanaro M, Bonanno C, Varotto L, Ometto R, Vincenzi M. Left ventricular versus biventricular dysfunction in idiopathic dilated cardiomyopathy. Am J Cardiol 1999;83:120-2,A9.

16. Gopinathannair R, Etheridge SP, Marchlinski FE, Spinale FG, Lakkireddy D, Olshansky B. Arrhythmiainduced cardiomyopathies: Mechanisms, recognition, and management. J Am Coll Cardiol 2015;66:1714-28.

17. Liang JJ, Silvestry FE. Mechanistic insights into mitral regurgitation due to atrial fibrillation: "Atrial functional mitral regurgitation". Trends Cardiovasc Med 2016;26:681-9.

18. Silbiger JJ. Mechanistic insights into atrial functional mitral regurgitation: Far more complicated than just left atrial remodeling. Echocardiography 2019;36:164-9.

19. Enriquez-Sarano M, Avierinos JF, Messika-Zeitoun D, et al. Quantitative determinants of the outcome of asymptomatic mitral regurgitation. N Engl J Med 2005;352:875-83.

20. Koelling TM, Aaronson KD, Cody RJ, Bach DS, Armstrong WF. Prognostic significance of mitral regurgitation and tricuspid regurgitation in patients with left ventricular systolic dysfunction. Am Heart J 2002;144:524-9.

21. Benfari G, Antoine C, Miller WL, et al. Excess mortality associated with functional tricuspid regurgitation complicating heart failure with reduced ejection fraction. Circulation 2019;140:196-206.

22. Sugumar H, Nanayakkara S, Prabhu S, et al. Pathophysiology of atrial fibrillation and heart failure: Dangerous interactions. Cardiol Clin 2019;37:131-8.

23. Lau DH, Nattel S, Kalman JM, Sanders P. Modifiable risk factors and atrial fibrillation. Circulation 2017;136:583-96.

\section{Figure legends}

Figure 1 Study Design

Figure 2 Differences in Baseline Cardiac Remodeling Between Two Groups

— Mild, — Moderate, — Severe Valvular Regurgitation

— Mild, — Moderately Decreased RV Systolic Dysfunction

- LV Size Within Normal Limits 
+ Mild, ++ Moderate, +++ Severely Enlarged

Figure 3 Left- and Right-sided Chambers

(A) The left atrium was significantly more enlarged in the AF-CM group but the left ventricle was slightly more remodeled in the isolated CM group. The left ventricle was the outlier, being the only chamber more enlarged in CM group. The percentage of patients for left atrial and left ventricular enlargement are shown on the vertical axis. Mean dimensions are in $\mathrm{mL} / \mathrm{m}^{2}$ for left atrial volume index, and mean dimensions are in $\mathrm{mm}$ for left ventricular diastolic diameter.

(B) Moderate-to-severe enlargement of the right atrium was quite prevalent in the AF-CM phenotype and uncommon in $\mathrm{CM}$ not associated with $\mathrm{AF}$. Moderate-to-severe right ventricular enlargement and dysfunction were also more than twice as common in the AF-CM cohort.

Figure 4 Valvular Regurgitation

Moderate-to-severe mitral regurgitation was more common in the AF-CM group, but the difference was even more marked in the prevalence of moderate-to-severe tricuspid regurgitation.

Figure 5 Proposed Pathophysiological Mechanism Causing Phenotype of AF-CM

$\mathrm{BMI}=$ body mass index; $\mathrm{CAD}=$ coronary artery disease $\mathrm{HTN}=$ hypertension; $\mathrm{MR}=$ mitral regurgitation; $\mathrm{TR}=$ tricuspid regurgitation

Table 1 Demographic and clinical characteristics

\section{Characteristic}

AF-CM group $(\mathrm{n}=196)$

\section{CM group}

$(\mathrm{n}=197)$

p value

Age, years

$73.51+-12.14$

$64.18+-15.98$

$<0.001$

Body mass index

$31.5+-8.2$

$29.8+-7.7$

0.04

Sex, male

124 (63.3)

97 (49.2)

0.01

Race

$<0.001$

White 
$190(96.9)$

151 (76.7)

Black/African American

$4(2.0)$

39 (19.8)

Others

$2(1.0)$

7 (3.5)

Tobacco use

119 (60.7)

$132(67.0)$

0.19

Alcohol use

$24(12.2)$

$71(36.0)$

$<0.001$

Hypertension

138 (70.4)

$105(53.3)$

$<0.001$

History of PCI

36 (18.4)

38 (19.3)

0.82

Hyperlipidemia

115 (58.7)

$81(41.1)$

$<0.001$

Coronary artery disease

66 (33.7)

$41(20.8)$

$<0.01$

Diabetes mellitus

59 (30.1) 
$57(28.9)$

0.80

History of drug abuse

$4(2.1)$

$15(7.6)$

0.01

Outpatient diagnosis

$70(35.7)$

$20(10.2)$

$<0.001$

Presenting symptoms

$<0.001$

Congestive heart failure

$128(65.3)$

$82(41.6)$

Myocardial infarction

$0(0.0)$

46 (23.3)

Others/unknown

$68(34.7)$

$69(35.0)$

Mortality

$62(31.6)$

$44(22.3)$

0.04

Palpitations on presentation

23 (11.7)

$3(1.5)$

$<.0 .001$

LVEF on diagnosis

0.99

6- $40 \%$

43 (21.9)

42 (21.3) 
$31-35 \%$

40 (20.4)

$43(21.8)$

$26-30 \%$

$45(23.0)$

$43(21.8)$

$21-25 \%$

$33(16.8)$

$33(16.8)$

[?] $20 \%$

35 (17.9)

$36(18.3)$

Data presented as n (\%) or mean +- standard deviation.

$\mathrm{AF}=$ atrial fibrillation; $\mathrm{CM}=$ cardiomyopathy; $\mathrm{LVEF}=$ left ventricular ejection fraction; $\mathrm{PCI}=$ percutaneous coronary intervention.

Table 2 Echocardiogram Characteristics

\begin{tabular}{|c|c|c|c|c|}
\hline $\begin{array}{l}\text { Echocardiographic } \\
\text { characteristic }\end{array}$ & Total $(n=393)$ & $\begin{array}{l}\text { AF-CM group } \\
(\mathrm{n}=196)\end{array}$ & $\begin{array}{l}\text { CM group } \\
(\mathrm{n}=197)\end{array}$ & $\mathrm{p}$ value \\
\hline LVEF & $393,29.38 \pm 7.73$ & $196,29.54 \pm 7.68$ & $197,29.21 \pm 7.81$ & 0.67 \\
\hline $\begin{array}{l}\mathrm{LV} \text { diastolic } \\
\text { diameter }(\mathrm{mm})\end{array}$ & $381,5.32 \pm 0.82$ & $193,5.19 \pm 0.79$ & $188,5.45 \pm 0.84$ & 0.0024 \\
\hline LV size & & & & 0.0059 \\
\hline Normal/mild & $302(81.2)$ & $163(86.7)$ & $139(75.5)$ & \\
\hline Moderate/severe & $70(18.8)$ & $25(13.3)$ & $45(24.5)$ & \\
\hline RV size & & & & $<0.001$ \\
\hline Normal/mild & $320(83.1)$ & $145(75.5)$ & $175(90.7)$ & \\
\hline Moderate/severe & $65(16.9)$ & $47(24.5)$ & $18(9.3)$ & \\
\hline RV function & & & & $<0.001$ \\
\hline Normal/mild & $298(77.2)$ & $132(68.8)$ & $166(85.6)$ & \\
\hline Moderate/severe & $88(22.8)$ & $60(31.2)$ & $28(14.4)$ & \\
\hline LA size & & & & $<0.001$ \\
\hline Normal/mild & $127(41.9)$ & $35(24.6)$ & $92(57.1)$ & \\
\hline Moderate/severe & $176(58.1)$ & $107(75.4)$ & $69(42.9)$ & \\
\hline LA volume index & $334,45.1 \pm 18.4$ & $164,51.9 \pm 19.5$ & $170,38.5 \pm 14.5$ & $<0.001$ \\
\hline RA size & & & & $<0.001$ \\
\hline Normal/mild & $239(62.1)$ & $77(40.1)$ & $162(83.9)$ & \\
\hline Moderate/severe & $146(37.9)$ & $115(59.9)$ & $31(16.1)$ & \\
\hline $\begin{array}{l}\text { Mitral } \\
\text { regurgitation }\end{array}$ & & & & 0.001 \\
\hline Normal/mild & $211(58.9)$ & $92(50.6)$ & $119(67.6)$ & \\
\hline Moderate/severe & $147(41.1)$ & $90(49.4)$ & $57(32.4)$ & \\
\hline $\begin{array}{l}\text { Tricuspid } \\
\text { regurgitation }\end{array}$ & & & & $<0.001$ \\
\hline
\end{tabular}




\begin{tabular}{lllll}
\hline $\begin{array}{l}\text { Echocardiographic } \\
\text { characteristic }\end{array}$ & Total $(\mathbf{n = 3 9 3 )}$ & $\begin{array}{l}\text { AF-CM group } \\
(\mathbf{n = 1 9 6})\end{array}$ & $\begin{array}{l}\text { CM group } \\
(\mathbf{n = 1 9 7})\end{array}$ & p value \\
\hline Normal/mild & $225(63.7 \%)$ & $93(51.4 \%)$ & $132(76.7 \%)$ & \\
Moderate/severe & $128(36.3 \%)$ & $88(48.6 \%)$ & $40(23.3 \%)$ & \\
\hline
\end{tabular}

Data presented as $\mathrm{n}$, mean \pm standard deviation or $\mathrm{n}(\%)$.

AF-CM = cardiomyopathy with atrial fibrillation; $\mathrm{CM}=$ cardiomyopathy without dysrhythmia; LA, left atrial; $\mathrm{LV}=$ left ventricular; $\mathrm{LVEF}=$ left ventricular ejection fraction; $\mathrm{RA}=$ right atrial; $\mathrm{RV}=$ right ventricular.

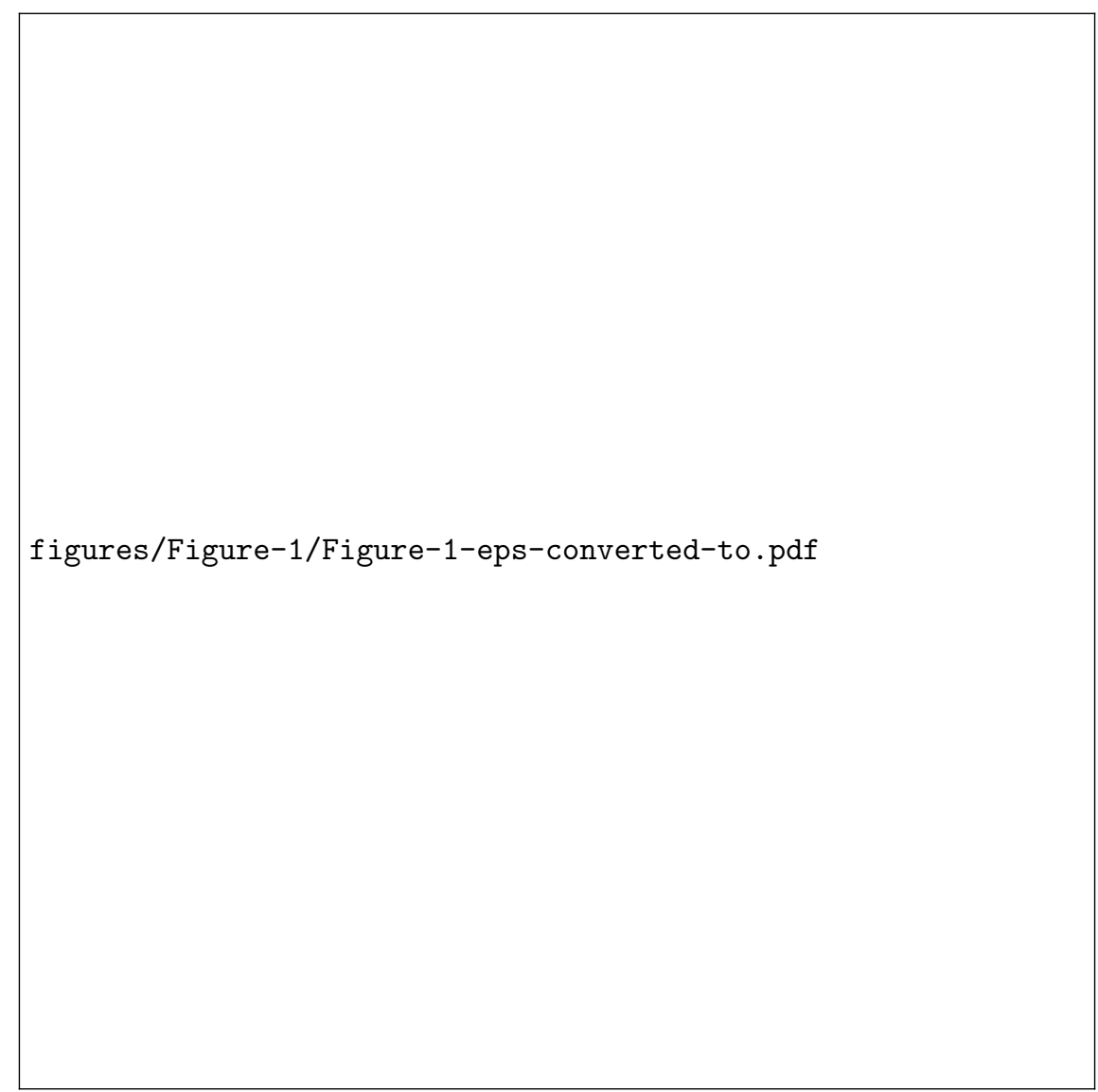




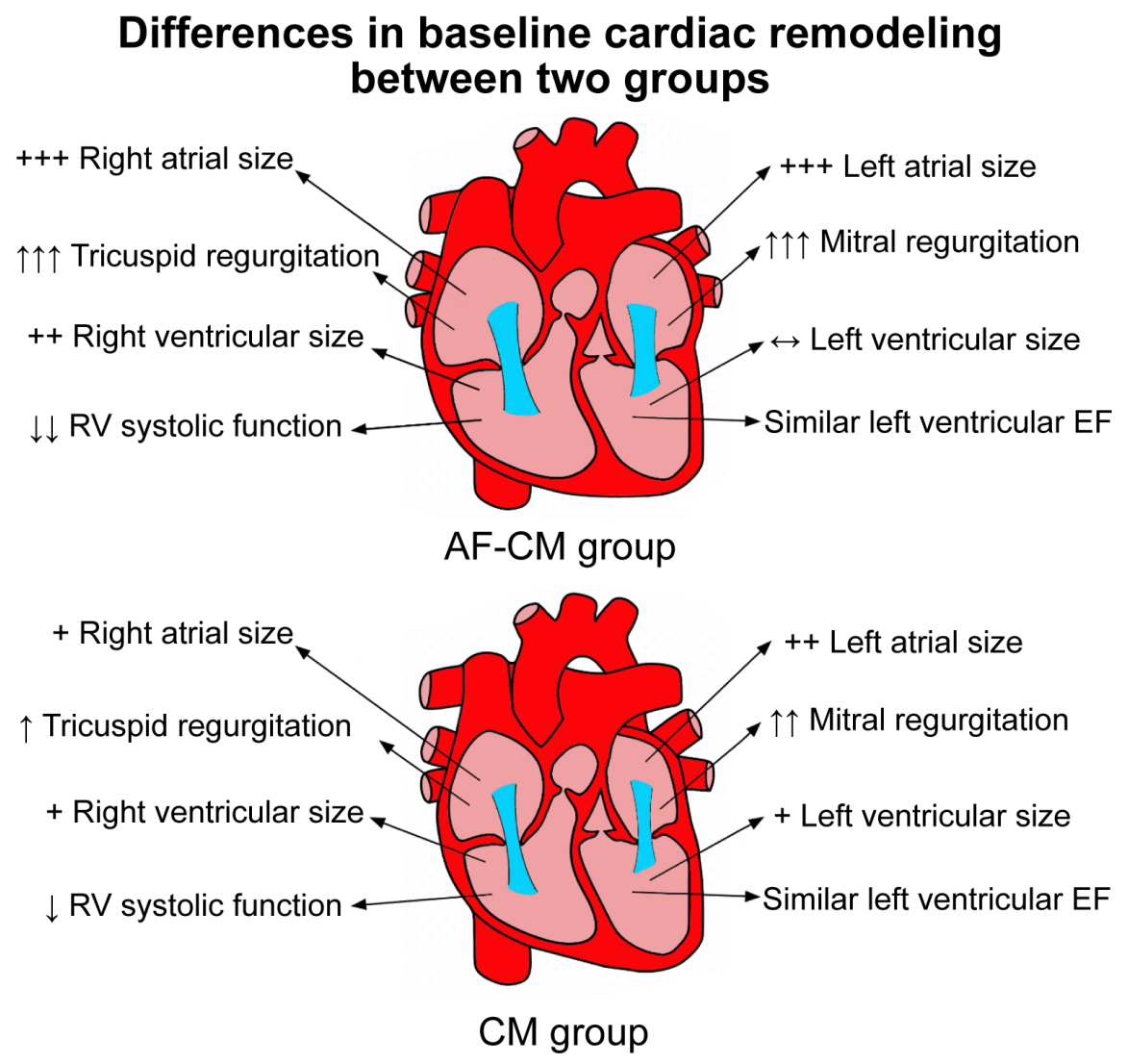



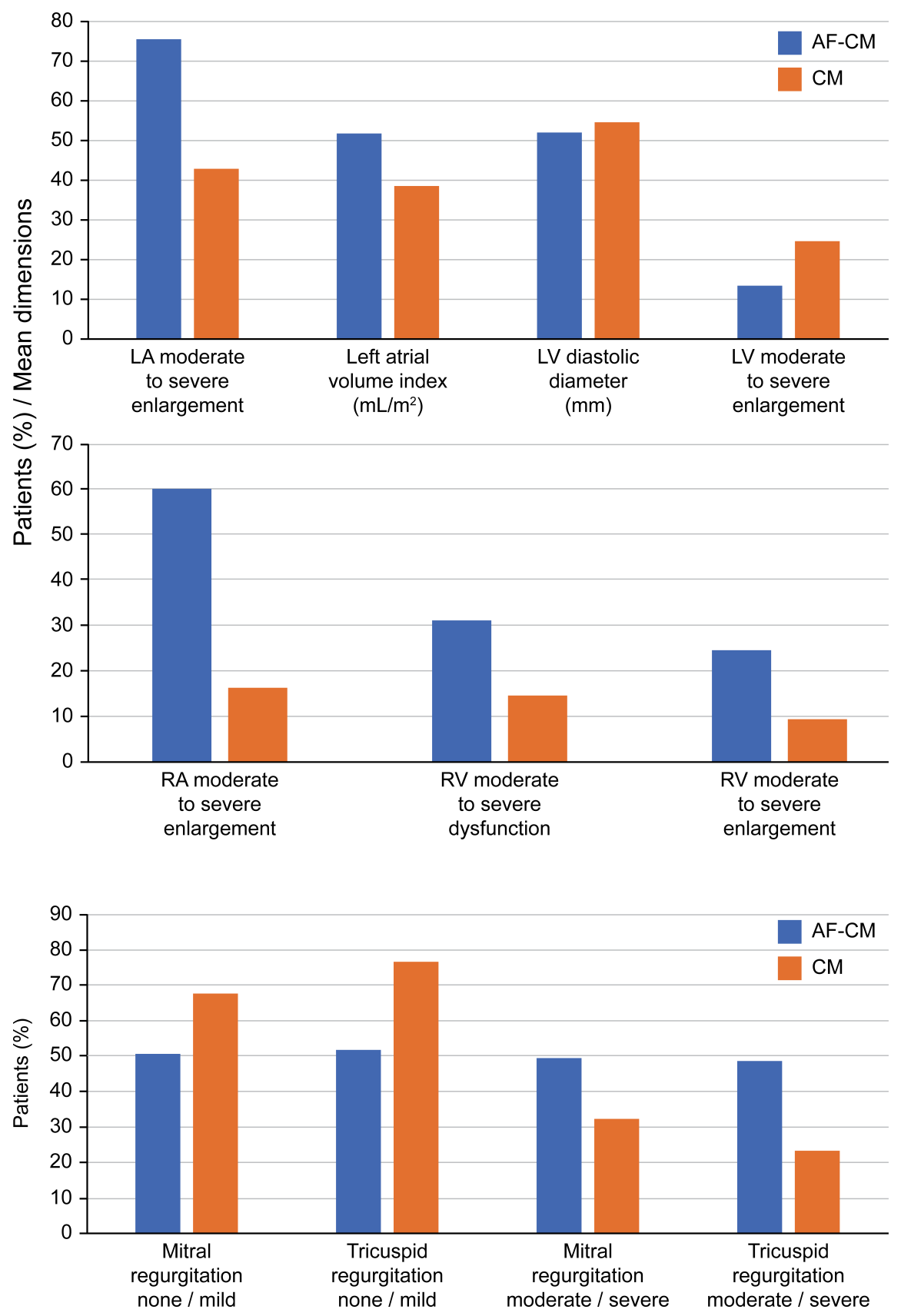


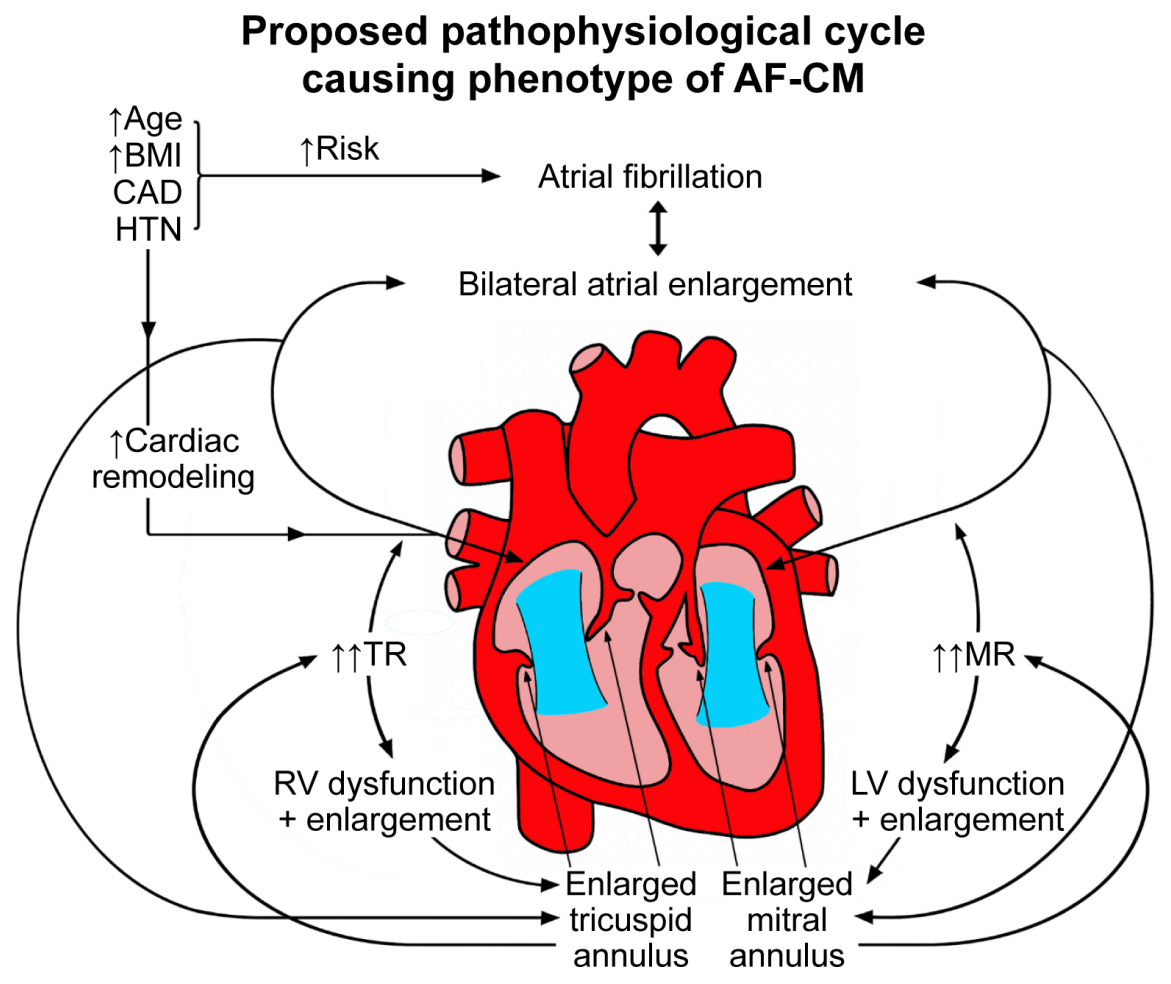

